\title{
IMMERSED CODIMENSION ONE PROJECTIVE SPACES IN SPHERICAL SPACE FORMS
}

\author{
J. SCOTT CARTER
}

(Communicated by Fredrick R. Cohen)

\begin{abstract}
Let a finite group of order $2 k$ act freely on an $n$-sphere. Then there is a generic immersion of an $(n-1)$-dimensional projective space in the quotient space; the induced homomorphism on the fundamental group is injective. Any such immersion has at least $\left(\begin{array}{l}k \\ n\end{array}\right) \frac{1}{k} \quad n$-tuple points. A better lower bound is also given.
\end{abstract}

In [1] an answer to a question that was posed to me by John Wood was presented. Specifically, any generic substantial immersion of a projective plane in a 3-dimensional lens space of type $(2 k, q)$ must have at least $\left(\begin{array}{l}k \\ 3\end{array}\right) \frac{1}{k}$ triple points. The appropriate generalization of that result is contained herein.

Suppose that a finite group $G$, of order $2 k$ acts freely and properly discontinuously on $S^{n}$. Then Milnor's condition [2] gives a unique element of order 2 that is necessarily central. Let $G$ denote the quotient group $G /(\mathbf{Z} / 2)$. Let

$$
\left\{\left(\begin{array}{l}
G \\
r
\end{array}\right)\right\}=\left\{\left\{g_{1}, \ldots, g_{r}\right\} \subset G: g_{i} \neq g_{j}\right\} \text {. }
$$

Then $G$ acts on $\left\{\left(\begin{array}{l}G \\ r\end{array}\right)\right\}$ as follows:

$$
\left\{g_{1}, \ldots, g_{r}\right\} g:=\left\{g_{1} g, \ldots, g_{r} g\right\} .
$$

Let $B(G, r)$ denote the number of orbits under this action.

All maps are smooth; all immersions are to be put into general position. The $n$-tuple point manifold of such an immersion is 0-dimensional and thus forms a discrete set. The main result can now be stated.

Theorem 1. Any generic immersion $f: \mathbf{R} P^{n-1} \rightarrow S^{n} / G^{\prime}$ such that $\pi_{1}(f) \neq 0$ has at least $B(G, n)$ n-tuple points.

The number of orbits, $B(G, r)$, of the group $G$ acting on its $r$-fold subsets is computed below in Theorem 3 .

Proof. The induced homomorphism, $\pi_{1}(f)$, on the fundamental group is an injection. Thus there is an element of order 2 in $G$; this involution must

Received by the editors March 28, 1988.

1980 Mathematics Subject Classification (1985 Revision). Primary 57S25; Secondary 57R42, 45,95 . 
be central by [2]. The covering $S^{n} \rightarrow S^{n} / G^{\prime}$ factors through a homotopy $\mathbf{R} P^{n}$. The map $f$ lifts to $k$ distinct lifts $f_{g}: \mathbf{R} P^{n-1} \rightarrow$ " $\mathbf{R} P^{n}$ " where $g \in$ $G=G^{\prime} /(\mathbf{Z} / 2)$. Each such lift is injective on the fundamental group, and consequently $f_{g}\left(\mathbf{R} P^{n-1}\right)$ represents a nonzero $(n-1)$-dimensional $\bmod 2$ homology class. Therefore, the transverse intersection, $\bigcap_{j=1}^{n} f_{g_{j}}\left(\mathbf{R} P^{n-1}\right)=Z\left\{g_{1}, \ldots, g_{n}\right\}$, is nonempty and contains an odd number of points. For each lift of $f$ is dual to the 1-dimensional cohomology generator. The quotient group $G$ acts on $\left\{Z\left\{g_{1}, \ldots, g_{n}\right\}: g_{i} \in G\right\}$ by right translation. There are $B(G, n)$ orbits of this action. Since each set $Z\left\{g_{1}, \ldots, g_{n}\right\}$ contains at least one point there are at least $B(G, n) n$-tuple points. This completes the proof.

Corollary 2. Let $f: \mathbf{R} P^{n-1} \rightarrow S^{n} / G$ be a generic immersion such that $\pi_{1}(f)$ is injective. Then $f$ has at least $\left(\begin{array}{l}k \\ n\end{array}\right) \frac{1}{k}$ n-tuple points.

Proof. The orbit of each set $Z\left\{g_{1}, \ldots, g_{n}\right\}$ has at most $k$ elements. Therefore, there are at least $\left(\begin{array}{l}k \\ n\end{array}\right) \frac{1}{k} \quad n$-tuple points of $f$.

Theorem 3. The number of orbits, $B(G, r)$, of the right $G$ action, $\left\{\left(\begin{array}{c}G \\ r\end{array}\right)\right\} \times G \rightarrow$ $\left\{\left(\begin{array}{l}G \\ r\end{array}\right)\right\}$, is given by the formula

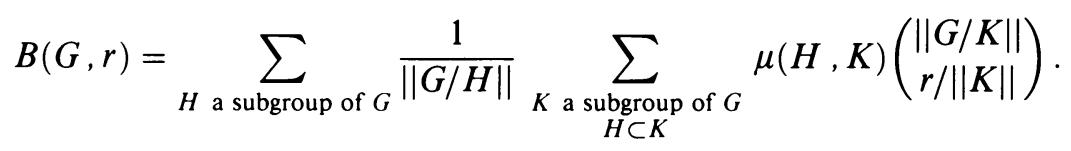

Here $\mu$ is the Mobius function associated to the partially ordered set of subgroups of $G$ (see [3]).

Proof. Let $x \in\left\{\left(\begin{array}{l}G \\ r\end{array}\right)\right\}$ and suppose that $H$ is a subgroup of $G$ that is contained in the stabilizer of $x$. Then $x$ can be written as a disjoint union of cosets: $x=g_{1} H \cup \cdots \cup g_{d} H$ where $d\|H\|=r$. Thus the number of elements $x$ such that $H$ is contained in the stabilizer of $x$ is equal to $\left(\begin{array}{c}\|G / H\| \| \\ r /\|H\|\end{array}\right)$. This choice function is meant to be equal to zero if $r /\|H\|$ is not an integer. By the Mobius inversion formula [3], the number of elements $x$ such that the stabilizer of $x$ is equal to $H$ is given by the expression:

$$
\sum_{\substack{K \text { subgroup of } G \\
H \subset K}} \mu(H, K)\left(\begin{array}{c}
\|G / K\| \\
r /\|K\|
\end{array}\right) .
$$

To obtain the number of orbits multiply this expression by $\|G / H\|^{-1}$ and sum over all subgroups $H$ of $G$. This completes the proof.

Suppose that $G^{\prime}$ is a group acting on the $n$-sphere that satisfies Milnor's condition (every element of order 2 is central), and suppose that $G$ is of order $2 k$. Then $S^{n} / G^{\prime}$ is covered by a homotopy $\mathbf{R} P^{n}$. There is an immersion of $\mathbf{R} P^{n-1}$ into this fake $\mathbf{R} P^{n}$ by a characteristic classes computation. The composition $\mathbf{R} P^{n-1} \rightarrow " \mathbf{R} P^{n} " \rightarrow S^{n} / G$ is an immersion. The fundamental 
group of $\mathbf{R} P^{n-1}$ injects in $G^{\prime}$. In summary, there is the following:

Every finite group, $G^{\prime}$, of order $2 k$ that acts freely on $S^{n}$ yields an immersion of $\mathbf{R} P^{n-1}$ in $S^{n} / G^{\prime}$ that has at least $B(G, n) n$ tuple points.

Realizing $B(G, n)$ as the number of $n$-tuple points of an immersed projective space is subtle even in the case of 3-dimensional space forms. For example, there is an immersion of $\mathbf{R} P^{2}$ in the lens space $L(8,3)$ with exactly one triple point, but the minimum number of triple points that can be achieved in $L(8,1)$ is 3 . To see that no fewer than 3 can be achieved is not difficult:

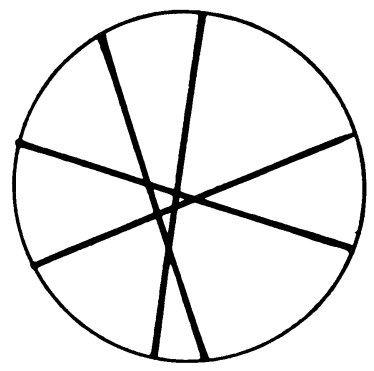

Figure 1

Consider an immersion of $\mathbf{R} P^{2}$ into $L(8,1)$. In a disk transverse to the core of the 1-handle of the standard solid torus decomposition of the lens space, the projective plane appears as intersecting arcs. These arcs are depicted in the figure below. Four chords in the unit disk are in general position and connect antipodal points of the unit circle. After this figure undergoes a rotation of $\pi / 4$, the intersection pattern that appears among the chords has changed. The chords may be isotoped with their end points fixed until the original intersection pattern appears. Independent of the original intersection pattern, there are at least 3 triple points during the isotopy.

It is often easy to construct $G$ invariant immersions of $k$ copies of $\mathbf{R} P^{n-1}$ in $\mathbf{R} P^{n}$, but these are usually not in general position. Placing them in general position with exactly $\left(\begin{array}{l}k \\ n\end{array}\right) \quad n$-tuple points can yield immersions that are not equivarint. To obtain equivariant general position immersions the action of $G$ on the multiple point manifolds $Z\left\{g_{1}, \ldots, g_{n}\right\}$ must be free.

A result in the positive direction is given in [1]. In the 3-dimensional lens space $L(2 k, q)$ there are immersions with exactly $q\left(\begin{array}{c}k-q \\ 2\end{array}\right)+\left(\begin{array}{l}q \\ 3\end{array}\right)$-triple points. A case by case analysis as suggested above determines whether this number is minimal.

The author gratefully acknowledges the help of John Moody who pointed out that orbits could be counted by means of a Mobius inversion. 


\section{BIBLIOGRAPHY}

1. J. Scott Carter, Immersed projective planes in lens spaces, Preprint.

2. J. Milnor, Groups which act on $S^{n}$ without fixed points, Amer. J. Math. 79 (1957), 623-630.

3. Gian-Carlo Rota, On the foundation of combinatorial theory I. Theory of Mobius functions, Z. Wahrsch. Verw. Gebiete 2 (1968), 340-368.

Department of Mathematics, University of Texas, Austin, Texas 78712

Current address: Department of Mathematics, Wayne State University, Detroit, Michigan 48202 